DOI: https:/doi.org/10.32789/publichealth.2021.1010

\title{
Presumed Ocular Tuberculosis - Challenges in Diagnosis
}

\author{
Sarah Sathyapriya ${ }^{1,2}$, Loh Chow Chin ${ }^{2}$, Wan Hazabbah Wan Hitam ${ }^{1}$ \\ ${ }^{I}$ Department of Ophthalmology and Visual Science, School of Medical Sciences, Universiti Sains Malaysia, \\ 16150 Kubang Kerian, Kelantan, Malaysia,sarahsathya238@gmail.com,hazabbah@usm.my, \\ hazabbah@yahoo.com \\ 2Ophthalmology Clinic, Hospital Sultanah Bahiyah, Malaysia,flcc10@yahoo.com
}




\title{
PRESUMED OCULAR TUBERCULOSIS - CHALLENGES IN DIAGNOSIS
}

\begin{abstract}
Mycobacterium tuberculosis has a large spectrum of extra-pulmonary manifestations, and ocular tuberculosis is one of them. Diagnosis of ocular tuberculosis is often presumptive due to its extreme variability of ocular manifestations and difficulty isolating the organism through biopsy or culture. We report three cases of primary ocular tuberculosis with varied clinical presentations, namely neuroretintis, panuveitis, and occlusive vasculitis. Patients were aged 23 to 45 presented with symptoms of blurring of vision from 3 days to one week prior to presentation. Visual acuity ranged from $6 / 18$ to $2 / 60$. For all three cases, chest X-ray and serological investigation for infective causes were normal. Mantoux test and TB Quantiferon test were negative, and ESR was raised for the first two cases. Mantoux was positive, and ESR was not raised for the third case. Patients were diagnosed to have Presumed Ocular Tuberculosis. An anti-tubercular therapy was administered. There was significant improvement at post-initiation of medication. A high index of clinical suspicion is crucial due to the diverse clinical presentations of ocular tuberculosis. Early initiation of anti-tubercular therapy is vital for successful treatment.
\end{abstract}

Keywords: Extra-pulmonary TB, Mantoux test, Quantiferon, Ocular tuberculosis

\section{Introduction}

Ocular tuberculosis is an extra-pulmonary mycobacterial infection with variable manifestations as it can present in a manner similar to that of any type of intraocular inflammation (Albert et al., 2015). Routine clinical diagnosis is often presumptive due to its extreme variability of ocular manifestations and difficulty isolating the organism through biopsy or culture. This report is to describe three retrospective case series of presumed ocular tuberculosis with such variability in presentation treated successfully with anti-tubercular therapy in our ophthalmology department from 2019 to 2020.

\section{$\underline{\text { Case } 1}$}

A 45-year-old lady with underlying dyslipidemia presented with floaters in her right eye. It was associated with distortion of image for one day. She also had a history of high-grade intermittent fever one month prior to presentation. There was no history of blurring of vision, pain, or redness. She did not have any constitutional symptoms, prolonged cough, and high-risk behaviour. On examination, the visual acuity initially was 6/9 in both eyes. The right eye showed blurred optic disc margin over the temporal side, fluid extending from optic disc to maculo-papillary bundle with macula oedema. During subsequent follow-up, right eye visual acuity deteriorated to 2/60 with worsening of macula oedema. Funduscopy showed the presence of a small lesion over the edge of the optic disc with new choroidal lesions. She was initially diagnosed with neuroretinitis. Oral Azithromycin was started for her. After which, the lesion progressed to become granulomatous. She was investigated further for ocular tuberculosis. Chest X-ray was normal with Mantoux test $0 \mathrm{~mm}$. ESR was $112 \mathrm{~mm} / \mathrm{hr}$. Serological testing for VDRL, Toxoplasmosis, and Leptospira was negative. TB Quantiferon test was also negative. Fundus fluorescein angiography revealed a hot disc with few hyperfluorescent late staining areas at the superotemporal region. There was no capillary fallout area or vasculitis. A diagnosis of Presumed Ocular Tuberculosis was made. An anti-tubercular therapy of Isoniazid, Rifampicin, Pyrazinamide, and Levofloxacin was started. There was a significant clinical improvement after the initiation of treatment. The optic disc granuloma and choroidal lesions resolved. The macula star remained within for two months. The patient's visual outcome improved to $5 / 60$ pinhole $6 / 21$. 


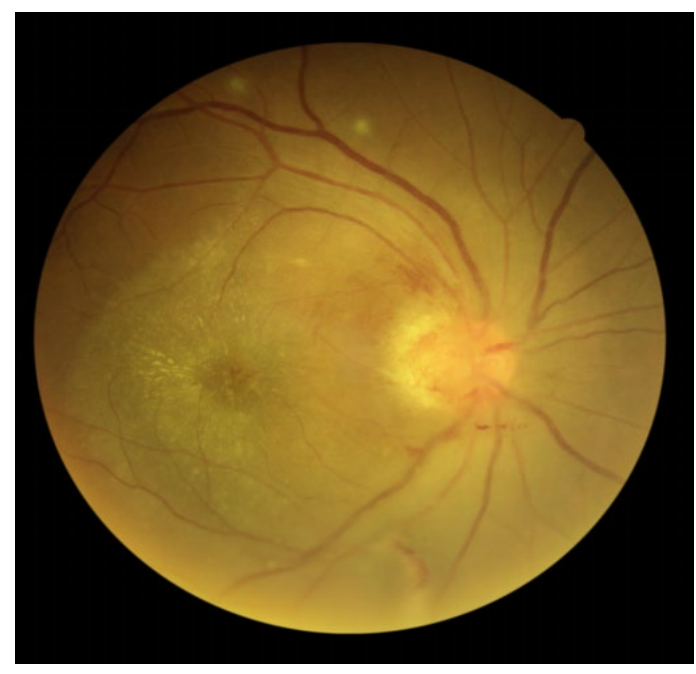

Figure 1: Presence of granulomatous lesion at temporal edge of the optic disc with macula oedema and early macular star

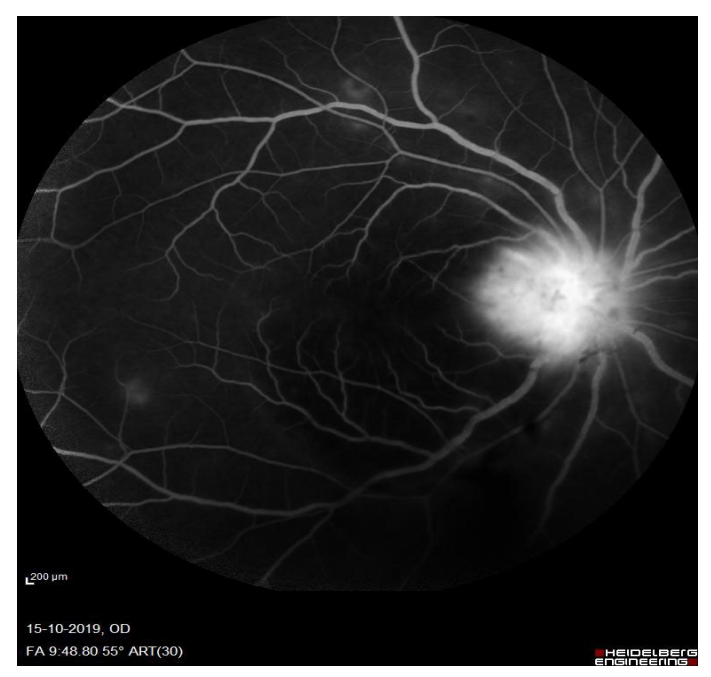

Figure 2: Fundus fluorescein angiography shows a hot disc with leakage of the dye in the disc area.

\section{Case 2}

A 37-year-old lady with no previous medical illness presented with a painless bilateral blurring of vision for three days. It was associated with fever for two weeks. There were no constitutional symptoms, prolonged cough, and high-risk behaviour. On examination, the visual acuity in the right eye was 6/36 pinhole was 6/36, and the left eye was counting fingers. Anterior segment examination of both eyes showed the presence of anterior uveitis. Funduscopy revealed a bilateral swollen disc with macula oedema and retinitis. There was a granulomatous lesion inferior to the left disc. Chest X-ray and Mantoux test were normal. ESR was $83 \mathrm{~mm} / \mathrm{hr}$. Serological tests for VDRL, Toxoplasmosis, and Leptospira were negative. TB Quantiferon test was also negative. Fundus fluorescein angiography showed leakage in both optic discs with focal staining at the superonasal region in the right eye and the superotemporal region in the left eye. The patient was diagnosed with 
Presumed Ocular Tuberculosis. She was started on an anti-tubercular therapy consisting of Isoniazid, Rifampicin, Pyrazinamide, and Ethambutol. There was a tremendous improvement after three months of treatment. Both optic discs swelling and retinitis resolved. The visual outcome also improved to $6 / 6$ in both eyes.

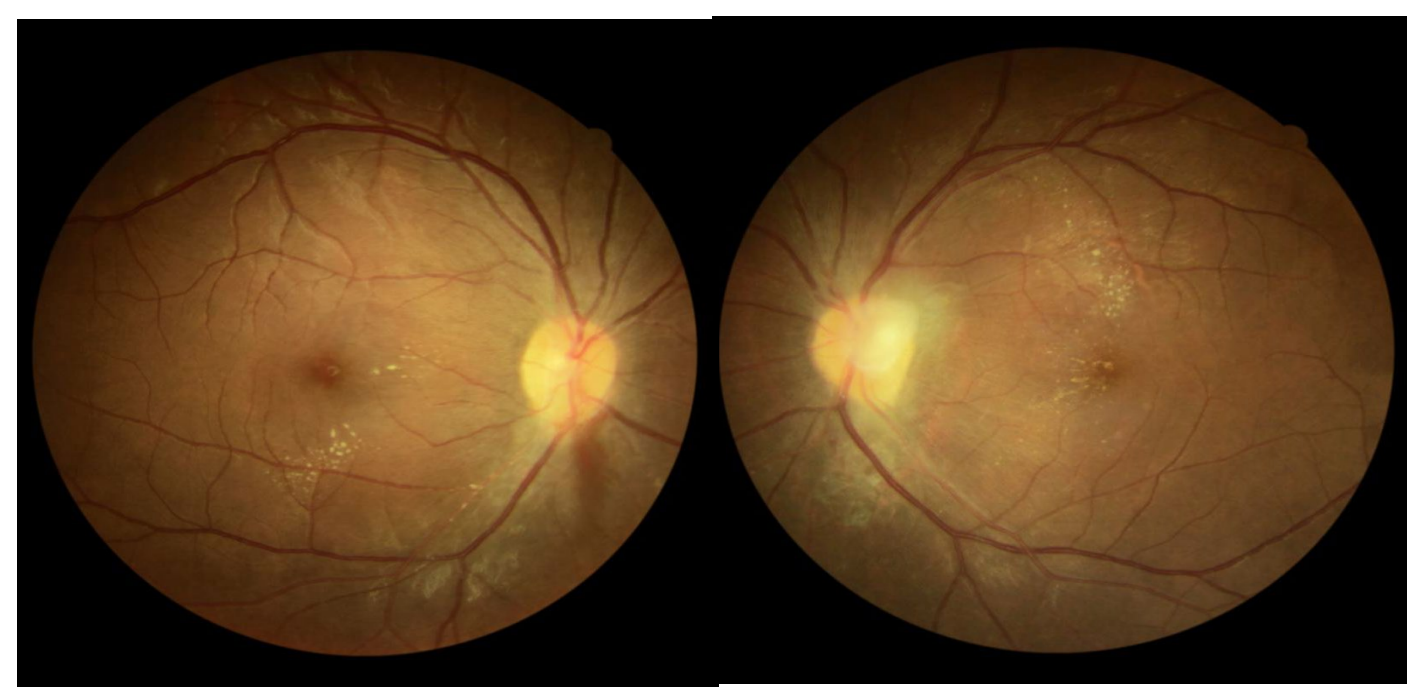

Figure 3: Bilateral optic discs swelling with macula oedema and retinitis. There is a granulomatous lesion inferior to the left disc.

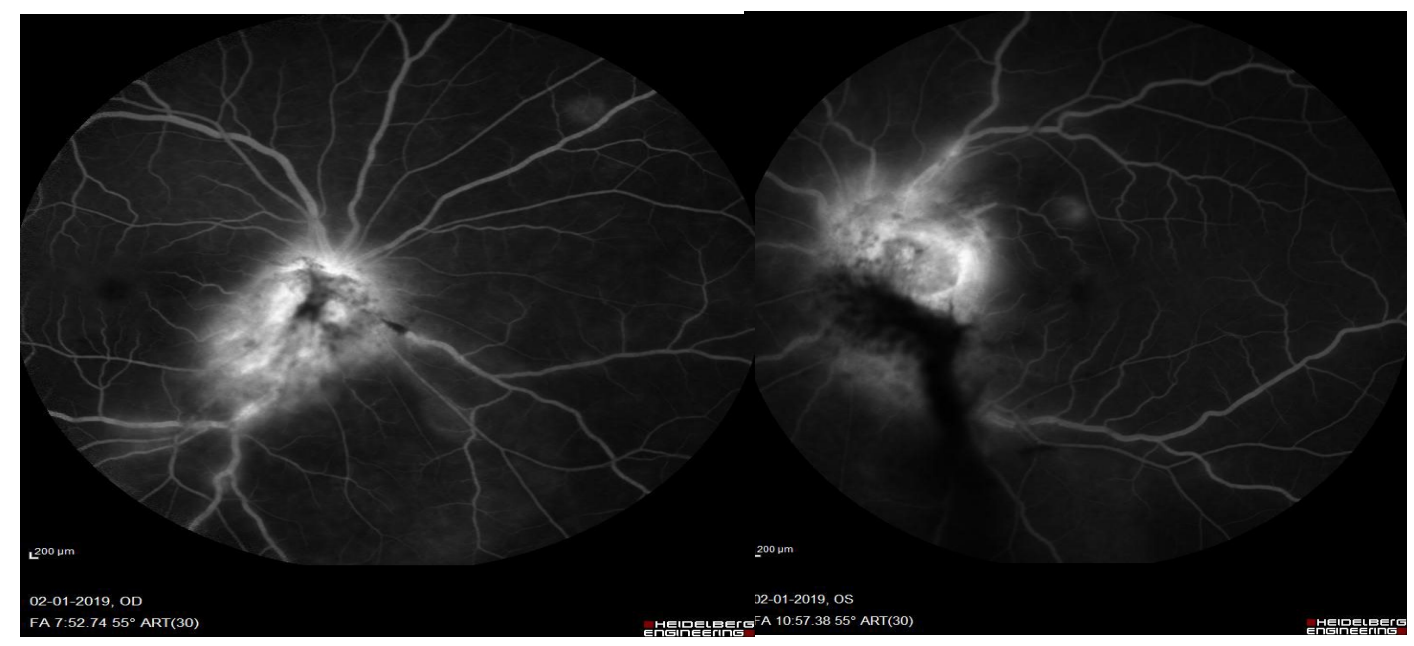

Figure 3: Fundus fluorescein angiography shows leakage at the optic disc with focal hyperfluorescent late staining lesion at right superonasal region and left superotemporal region. 


\section{Case 3}

A 23-year-old man with no medical illness presented with a painless bilateral blurring of vision for one week. There was no history of fever or prolonged cough. He did not have any constitutional symptoms and high-risk behaviour. On examination, the visual acuity in both eyes was 6/18. Funduscopy showed bilateral optic disc swelling with retinitis and vasculitis in superior and inferior arcuate. There was also macular oedema with retinal haemorrhages in all quadrants. The patient was investigated for bilateral eye occlusive vasculitis. Mantoux test was raised, measuring $20 \mathrm{~mm}$. ESR was $1 \mathrm{~mm} / \mathrm{hr}$. Chest X-ray was normal. Serological tests for VDRL and IgM Mycoplasma were negative. Fundus fluorescein angiography revealed a masking effect due to retinal haemorrhages with occlusive vasculitis and generalised capillary fall-out area. The patient was diagnosed to have ocular tuberculosis. An anti-tubercular therapy was started with oral corticosteroids. He also underwent multiple sessions of pan-retinal photocoagulations for both eyes. However, the course of healing was complicated by vitreous haemorrhages on the bilateral eye, for which the patient underwent a vitrectomy. Mycobacterium TB PCR taken was negative. The patient was a steroid responder thus developed secondary raised intraocular pressure that was controlled with topical intraocular pressure-lowering medications. Upon completing 9 months of anti-tubercular therapy (Isoniazid, Rifampicin, Pyrazinamide, and Ethambutol), the best-corrected visual acuity in the right eye was 6/12, and the left eye was 6/18. Both eyes were quiet. Fundus showed the presence of epiretinal membrane and gliosis inferior to disc in the left eye.

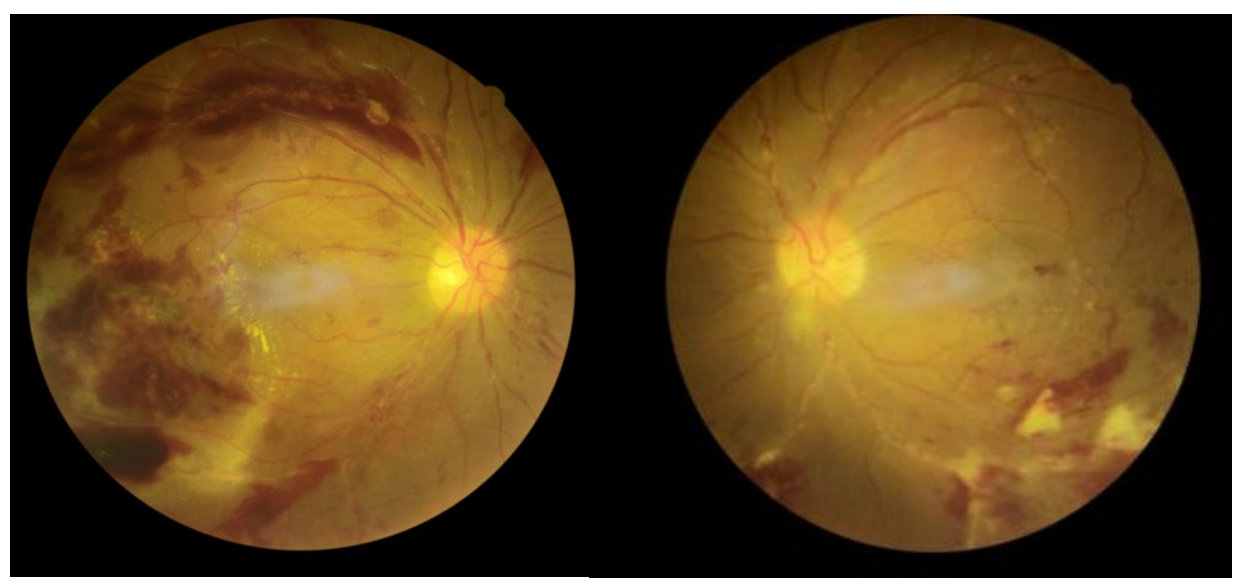

Figure 5: Bilateral diffuse retina haemorrhages and exudates with vasculitis in right superior and infero-temporal region and left the infero-temporal region. 


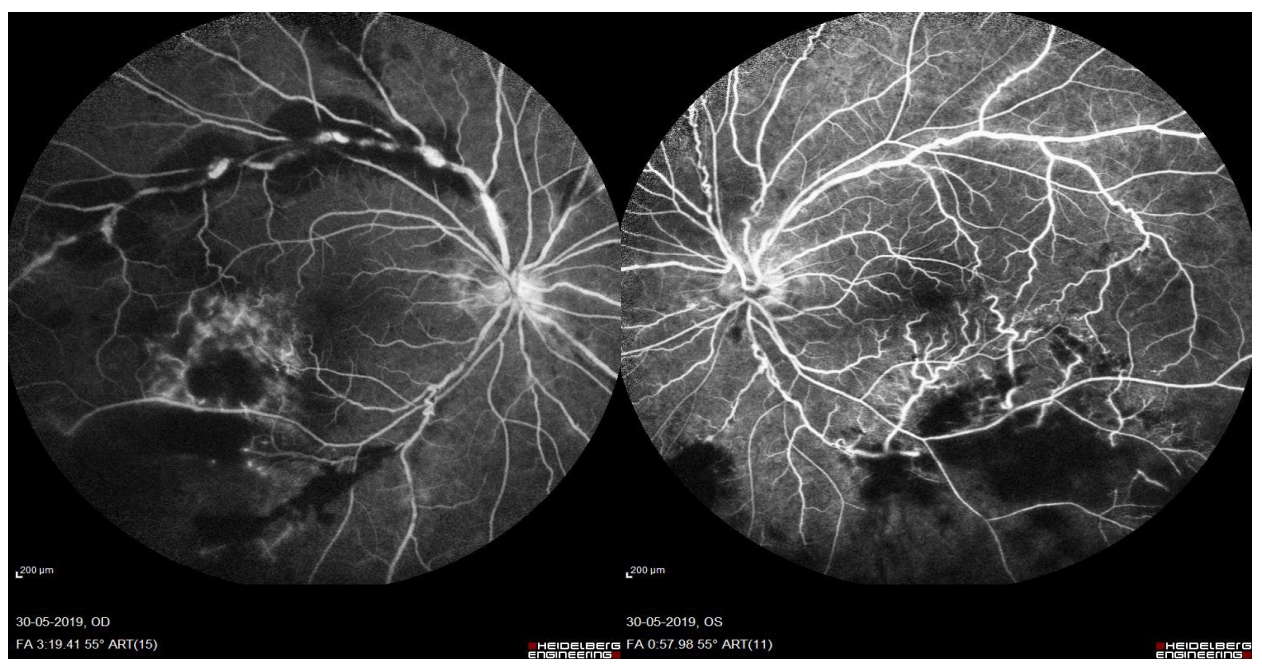

Figure 6: Fundus fluorescein angiography showing masking effect due to retinal haemorrhages with occlusive vasculitis and generalised capillary fall out area

\section{Discussion}

According to the World Health Organisation (WHO), an estimated 10.0 million people were affected by TB in 2018, with a mortality of 1.2 million among HIV-negative people (a 27\% reduction from 1.7 million in 2000) and an additional 251000 deaths among HIV-positive people. Up to $60 \%$ of patients with evidence of extrapulmonary TB may not have been diagnosed pulmonary TB (Albert et al., 2017). Ocular TB may be an initial presentation of extra-pulmonary dissemination of infection. It is important for all general practitioners, public health, and medical personnel to know the variable presentation of tuberculosis in the eye as many are still unaware of ocular TB and frequently misdiagnose ocular symptoms.

Ocular manifestations are possibly from direct mycobacterial invasion or may result from a delayed hypersensitivity response to mycobacterium (Shakarchi F.I et al., 2015 and Shahidatul-Adha et al., 2019). Primary ocular tuberculosis refers to the direct inoculation of Mycobacterium tuberculosis into the ocular surface with or without systemic involvement. In contrast, secondary ocular TB is defined as infection from a distant site like hematogenous spread via uveal and choroidal vasculature or contagious spread from adjacent structures, like the sinuses (Shakarchi F.I et al., 2015 and Yasaratne. B et al., 2010).

Ocular tuberculosis has various clinical presentations, and unfortunately, there are no pathognomonic ocular findings. In a study by Gupta, V. et al., 2007, in an observation among 158 patients diagnosed with presumed intraocular tuberculosis, posterior uveitis emerged as the most common (42\%), followed by anterior uveitis $36 \%$. Panuveitis was seen in $11 \%$ of the patients. These patients usually experience an insidious onset of symptoms that run a chronic course of redness and discomfort from weeks to months, followed by gradual disturbance of vision. Granulomatous uveitis of tuberculosis is often associated with mutton fat keratic precipitates, iris nodules, and posterior synechiae (Gupta, V. et al., 2007 and Gupta, A. et al., 2014).

Unlike uveitis, patients with tuberculous optic neuropathy, such as neuroretinitis, often have subtle initial symptoms such as floaters without any eye redness or pain, which rapidly deteriorate as observed in our first patient. Primary caregivers and general practitioners often disregard these subtle initial symptoms as there will be a very low index of suspicion of ocular tuberculosis. In a study involving the collection of data from the establishment of diagnosis in our patient posed many challenges. In all our patients, the timely initiation of anti- 
tubercular therapy was crucial for successful treatment. Although a positive therapeutic response is seen as hindsight support in our diagnosis, its inclusion in the diagnostic criteria, as in Gupta, V. et al., 2007, was essential in the treatment of our patient. Another classification of intraocular tuberculosis by Gupta, A. et al., 2014, a revised version of their guideline, which was done a decade prior, was proposed. This classification, however, excludes therapeutic success as one of the diagnostic criteria.

Based on a diagnostic guideline by Gupta A. et al., 2014, cases were divided into confirmed, presumed, and possible intraocular tuberculosis, as seen in Table 1.

Table 1 (below): Diagnostic criteria of ocular tuberculosis by Gupta, A. et al., 2014

Diagnostic Group

Confirmed IOTB

(both 1 and 2)

\section{Criteria}

2. Microbiological confirmation of (MTB) from ocular fluids/tissues

\section{Probable IOTB}

$(1,2$, and 3 together $)$
1. One clinical feature of IOTB (other etiologies excluded)

2. Radiological evidence of TB or extraocular TB signs

3. One of the following:

a. Documented exposure to TB

b. Immunological evidence TB infection

\section{Possible IOTB}

\section{$(1,2$, and 3 together $)$}

(or 1 and 4)
1. One clinical feature of IOTB (other etiologies excluded)

2. Radiologic evidence not consistent with $\mathrm{TB}$ infection and no extraocular TB extraocular TB

3. One of the following:

a. Documented exposure to TB

b. Immunological evidence TB infection

4. Radiological evidence of TB or extraocular TB signs but none of the characteristics given in 3 


\section{Conclusion}

These case series highlight the diverse clinical presentations of ocular tuberculosis in the absence of systemic tuberculosis. Ocular Tuberculosis involves almost all the ocular structures except the lens. It is difficult to isolate Mycobacterium tuberculosis through biopsy. Thus, diagnosis is based on clinical findings and suspicion of tuberculosis, especially in an endemic area. Newer diagnostic modalities, IGRA, and PCR aids in diagnosis. The importance of prompt initiation of anti-tubercular therapy is crucial for good clinical outcomes, as reported in all our 3 cases.

\section{References}

Agrawal, R., Gupta, B., Gonzalez-Lopez, J.J., Rahman, F., Phatak, S., Triantafyllopoulou, I., Addison, P.K., Westcott, M. and Pavesio, C.E., 2015. The role of anti-tubercular therapy in patients with presumed ocular tuberculosis. Ocular immunology and inflammation, 23(1), pp.40-46.

Albert, D.M. and Raven, M.L., 2017. Ocular tuberculosis. Tuberculosis and Nontuberculous Mycobacterial Infections, pp.313-330.

Babu K, Satish V, Satish S, Subbakrishna DK, Abraham MP, Murthy K.R., 2009. Utility of QuantiFERON TB gold test in a south Indian patient population of ocular inflammation. Indian J Ophthalmol. Nov-Dec; 57(6):427-30. doi: 10.4103/0301-4738.57147. PMID: 19861743; PMCID: PMC2812760.

Davis EJ, Rathinam SR, Okada AA, et al., 2012. Clinical spectrum of tuberculous optic neuropathy. $J$ Ophthalmic Inflamm Infect. ; 2(4):183-189. Doi: 10.1007/s12348-012-0079-5

Gupta, V., Gupta, A. and Rao, N.A., 2007. Intraocular tuberculosis - an update. Survey of ophthalmology, 52(6), pp.561-587.

Gupta A, Sharma A, Bansal R, Sharma K. 2015 Classification of intraocular tuberculosis. Ocul Immunol Inflamm. Feb; 23(1):7-13. doi: 10.3109/09273948.2014.967358. Epub 2014 Oct 14. PMID: 25314361.

Kanski, J.J. and Bowling, B., 2011. Clinical ophthalmology: a systematic approach. Elsevier Health Sciences.

Lyn, L.U., Nadras, I., Mee, Y.C., Nadarajah, G., Mohamed, S.O., Qamarruddin, F. and Hussein, A., 2019. Case Series of Ocular Tuberculosis; The Great Masquerader. Journal of Biomedical and Clinical Sciences (JBCS), 3(2), pp.1-5.

Nayak S, Basu S, Singh MK. Presumed tubercular retinal vasculitis with serpiginous-like choroid it is in the other eye. (2011) Ocul Immunol Inflamm. 19(5):361-2.

Shahidatul-Adha, M., Zunaina, E., Liza-Sharmini, A.T., Wan-Hazabbah, W.H., Shatriah, I., Mohtar, I., Azhany, Y. and Adil, H., 2017. Ocular tuberculosis in Hospital Universiti Sains Malaysia-A case series. Annals of medicine and surgery, 24, pp.25-30.

Shakarchi, F.I., 2015. Ocular tuberculosis: current perspectives. Clinical ophthalmology (Auckland, NZ), 9, p. 2223 .

Yasaratne, B., Madegedara, D., Senanayake, N. and Senaratne, T., 2010. A case series of symptomatic ocular tuberculosis and the response to anti-tubercular therapy. Ceylon Medical Journal, 55(1), pp.16-19. 